\title{
DISCUSSION
}

\section{Net pressure analysis of cantilever sheet pile walls}

\author{
R. A. DAY (1999). Géotechnique 49, No. 2, 231-245
}

K. S. Li, University of Hong Kong

The CIRIA report by Padfield \& Mair (1984) presents the so-called 'full' method and simplified method for analysis of cantilever retaining walls. The two methods are based on the pressure diagrams shown in Fig. 20. To correct for the approximations of the simplified method, Padfield \& Mair (1984) suggested that the critical embedment length obtained by the simplified method $d_{\mathrm{o}}$ should be increased by $20 \%$. That is to say, an estimate of the critical embedment length by the 'full' method, $d$, may be obtained using the relationship of $d=1 \cdot 2 d_{\mathrm{o}}$. For a cantilever retaining wall in a dry homogeneous soil, the ratio $d_{\mathrm{o}} / h$ at limiting equilibrium for the simplified method is given as (Padfield \& Mair, 1984)

$$
\frac{d_{\mathrm{o}}}{h}=\frac{1}{\sqrt[3]{\frac{K_{\mathrm{p}}}{K_{\mathrm{a}}}}-1}
$$

Equation (1) in the author's paper is in fact based on the simplified method described by Padfield \& Mair (1984). It is possible to obtain an exact solution for the 'full' method. The aim of this discussion is to present the results for the 'full' method and compare them with other methods discussed in the author's paper.

For a dry soil, the equations for force and moment equilibrium can be deduced by equating the area and first moment of area of various triangle formed by the pressure diagrams in Fig. 20(a) as follows:

(a) Force equilibrium:

$$
\Delta \mathrm{AOB}-\Delta \mathrm{ADC}=\Delta \mathrm{EFG}+\Delta \mathrm{EHO}
$$

where $\triangle \mathrm{AOB}=$ area of triangle $\mathrm{AOB}$ and so on

(b) Moment equilibrium about point ' $\mathrm{O}$ ':

$$
\begin{aligned}
z_{\mathrm{AOB}} & \times \Delta \mathrm{AOB}-z_{\mathrm{ADC}} \times \Delta \mathrm{ADC}=z_{\mathrm{EFG}} \\
& \times \Delta \mathrm{EFG}+z_{\mathrm{EHO}} \times \Delta \mathrm{EHO}
\end{aligned}
$$

where $z_{\mathrm{AOB}}=$ moment arm of triangle $\mathrm{AOB}$ about point $\mathrm{O}$ and so on.

The two unknowns in the above system of equations are $d$ and $d_{\mathrm{f}}$ as defined in Fig. 20(a). Based on linear variation of earth pressure distributions, the following expressions are obtained:

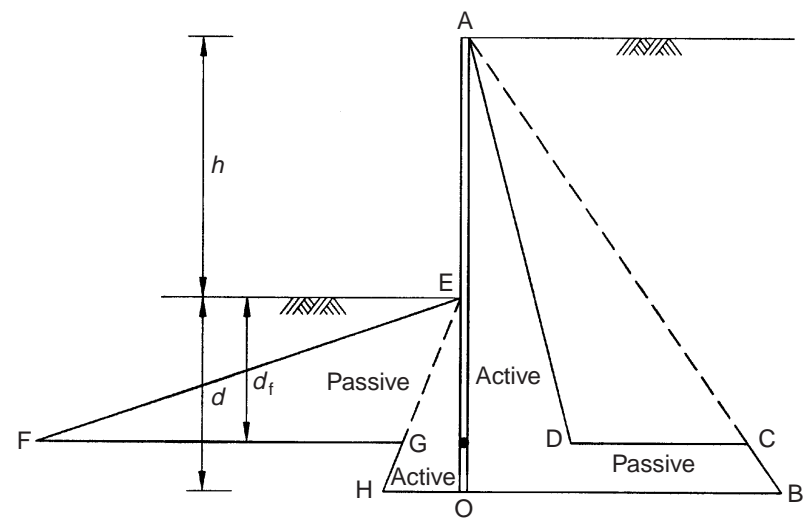

(a)

$$
\begin{aligned}
& \mathrm{DC}=\left(K_{\mathrm{p}}-K_{\mathrm{a}}\right) \gamma\left(h+d_{\mathrm{f}}\right) \\
& \mathrm{FG}=\left(K_{\mathrm{p}}-K_{\mathrm{a}}\right) \gamma d_{\mathrm{f}} \\
& \mathrm{OB}=K_{\mathrm{p}} \gamma(h+d) \\
& \mathrm{OH}=K_{\mathrm{p}} \gamma d
\end{aligned}
$$

The moment arms of the force triangles about point ' $\mathrm{O}$ ' are expressed as follows:

$$
\begin{aligned}
& z_{\mathrm{AOB}}=\frac{1}{3}(h+d) \\
& z_{\mathrm{ADC}}=\left(d-d_{\mathrm{f}}\right)+\frac{1}{3}\left(h+d_{\mathrm{f}}\right) \\
& z_{\mathrm{EFG}}=\left(d-d_{\mathrm{f}}\right)+\frac{1}{3} d_{\mathrm{f}} \\
& z_{\mathrm{EHO}}=\frac{1}{3} d
\end{aligned}
$$

Utilising the above relationships and introducing the dimensionless ratios $k=K_{\mathrm{p}} / K_{\mathrm{a}}, \alpha=d_{\mathrm{f}} / d$ and $r=d / h$, equations (11) and (12) can be simplified to yield the following two equations respectively:

$$
\begin{aligned}
& \alpha^{2} r^{2}+\alpha r+\frac{r^{2}-k(1+r)^{2}+(k-1)}{2(k-1)}=0 \\
& k(1+r)^{3}-(k-1)(1+\alpha r)^{2}(1+3 r-2 \alpha r) \\
& \quad=(k-1) \alpha^{2} r^{2}(3 r-2 \alpha r)+r^{3}
\end{aligned}
$$

Equation (15) is a quadratic equation in $\alpha r$. The equation can be solved to yield the following solution of $\alpha r$ in terms of $k$ and $r$ :

$$
\alpha r=\frac{-1+\sqrt{1-2 c}}{2}
$$

where

$$
c=1+\frac{r^{2}-k(1+r)^{2}}{k-1}
$$

By substituting equation (17) into equation (16), the latter equation becomes a non-linear equation of two variables only, namely $r$ and $k$. For a given value of $k$, the solution of $r$ can be obtained easily by iteration. Once the value of $r$ is obtained, the value of $h / d$ is simply given as the reciprocal of $r$.

Using the above procedures, the values of $h_{\mathrm{c}} / d$ for different values of $k$ can be computed. Fig. 21 compares the variation of

Fig. 20. Pressure distribution based on: (a) full method; (b) simplified method

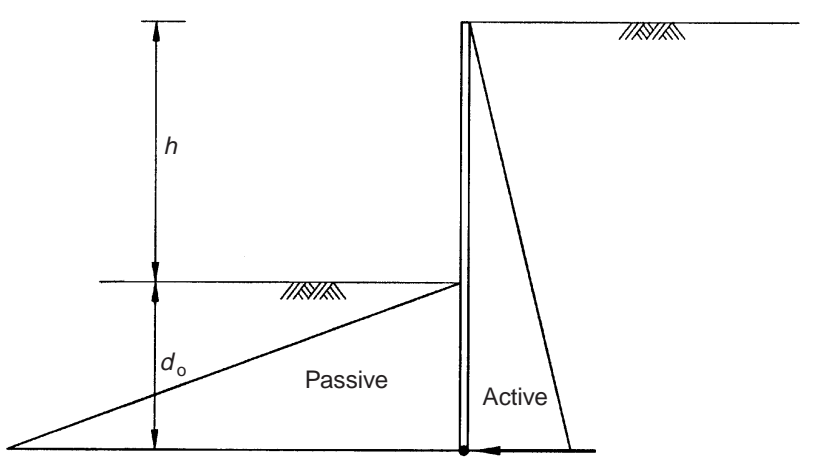

(b) 


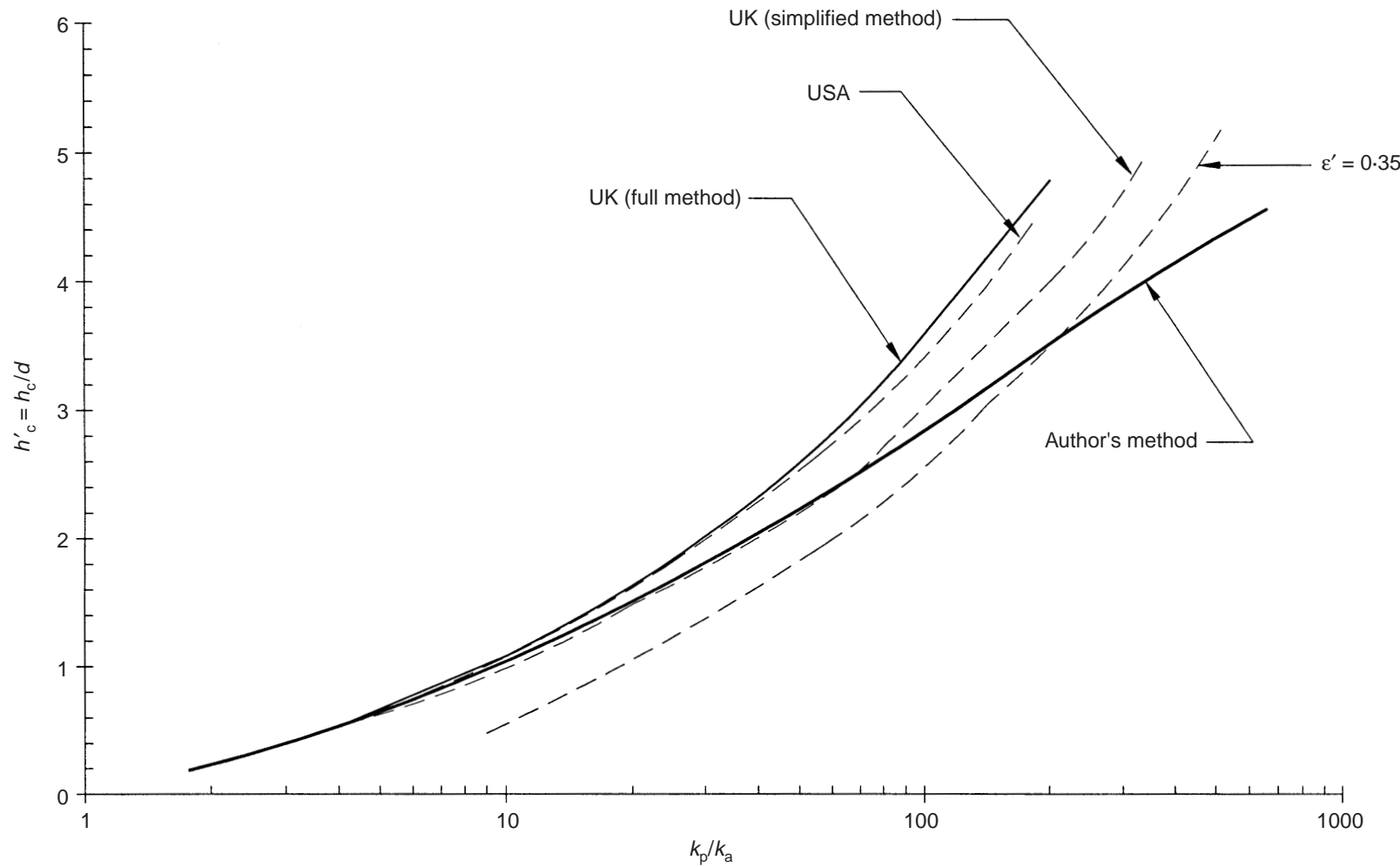

Fig. 21. Comparison of design methods

$h_{\mathrm{c}} / d$ for the 'full' method with other methods discussed by the author. The 'full' method gives results very similar to the US method. It is interesting to observe that the simplified method gives answers closer to that obtained by the author's method. If the author's method does represent a solution closer to the true behaviour of a cantilever retaining wall, the simplified method is in fact more accurate than the 'full' method.

It appears that most of the comparative studies published in the literature on the critical embedment lengths of a cantilever retaining wall are confined to a dry soil. It will be useful if the author can extend his study and present some additional results for a cantilever retaining wall that retains a water table behind it.

\section{Author's reply}

The author appreciates the comments made by Professor Li, and welcomes his contribution concerning the UK full method. As highlighted by Professor Li the UK full method gives results very similar to the US method. The reason for this is revealed by examination of the net pressure distributions. Common assumptions made by both methods are:

(a) Active pressure is fully mobilised from the top of the wall to some point considerably below the excavation level.

(b) Passive pressure is fully mobilised immediately below excavation level.

(c) At the bottom of the wall the net pressure is equal to the maximum possible value:

$$
p_{2}=\gamma(h+d) K_{\mathrm{p}}-\gamma d K_{\mathrm{a}}
$$

Figure 22 compares the net pressure distributions assumed by the US and UK full method for a wall with the same excavation height. The assumed pressure distributions are quite similar. They differ only in the lower $7 \%$ of the wall. The resulting difference in the calculated critical excavation depth given by the US method ( $h_{\mathrm{c}}^{\prime}$ USA) and the UK full method ( $h_{\mathrm{c} F U L L}^{\prime}$ ) is

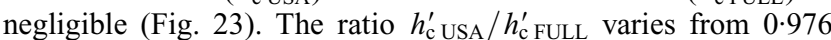
when $K_{\mathrm{p}} / K_{\mathrm{a}}=3$ to 0.991 when $K_{\mathrm{p}} / K_{\mathrm{a}}=500$.

The UK full method also provides a framework of reference for the UK simplified method. Using the common assumption that $d / d_{\mathrm{o}}=1.2$ the results of the UK simplified method are

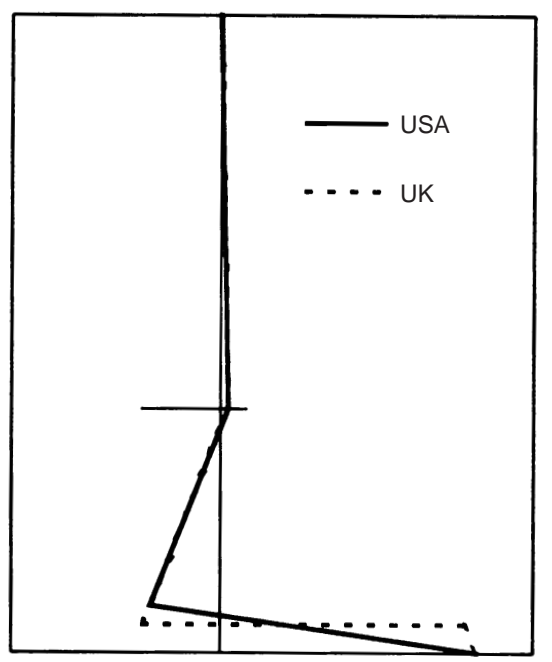

Fig. 22. Net pressure distributions

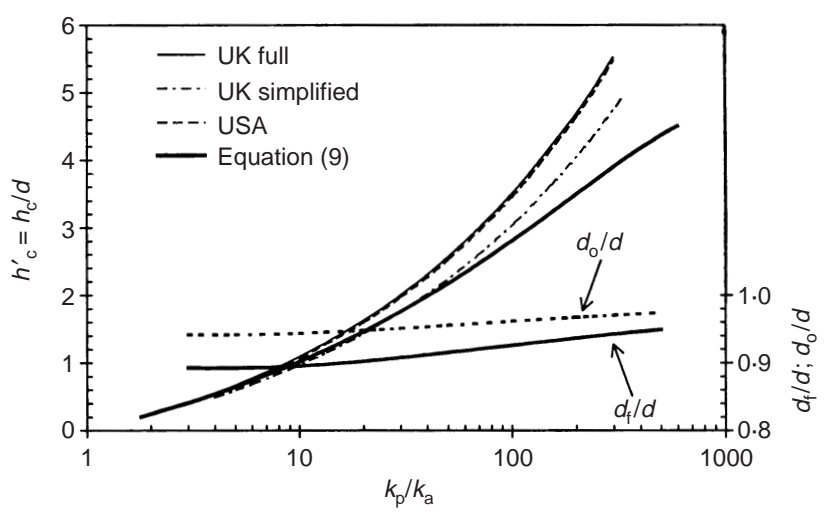

Fig. 23. Comparison of methods 
plotted on Fig. 23. The UK simplified method gives values of $h_{\mathrm{c}}^{\prime}$ between 0.86 and 0.88 of those for the UK full method.

The UK full method provides the value of $\alpha=d_{\mathrm{f}} / d$. Using equation (1) and the solution to the UK full method, we can also obtain a relationship for $d_{\mathrm{o}} / d$ :

$$
\frac{d_{\mathrm{o}}}{d}=\frac{h_{\mathrm{c} \text { FULL }}^{\prime}}{\sqrt[3]{K_{\mathrm{p}} / K_{\mathrm{a}}}-1}
$$

These two ratios are also plotted on Fig. 23. It can be seen that $d_{\mathrm{o}} / d$ is about midway between $d_{\mathrm{f}} / d$ and $1 \cdot 0$. this confirms that $d_{\mathrm{o}}$ is approximately midway between $d_{\mathrm{f}}$ and $d$ (Padfield \& Mair, 1984). Also (since $0.94<d_{\mathrm{o}} / d<0.97$ ) the depth, $d$, obtained from the UK full method is between $3 \%$ and $6 \%$ greater than $d_{\mathrm{o}}$. In order to obtain the UK full solution by using the UK simplified method an increase to $d_{0}$ of only $3-6 \%$ is required. The more common increase of $20 \%$ provides embedment depths between $13 \%$ and $17 \%$ greater than the UK full method.

In summary:

(a) The assumed net pressure distributions used in the US and the UK full method are identical except for very near to the bottom of the wall. For practical purposes the corresponding critical excavation depths are equal.

(b) The difference between the UK full method and the UK simplified method is due to the conservative assumption that $d / d_{\mathrm{o}}=1 \cdot 2$. However, it appears that this assumption may indeed give a solution closer to the real behaviour observed in centrifuge model tests and finite element analyses. 